\title{
ON COMPACT FIBERINGS OF THE PLANE
}

\author{
GAIL S. YOUNG
}

In a recent paper [2], ${ }^{1}$ Montgomery and Samelson have raised the question whether there exists, for some $n$, a compact fibering of Euclidean $n$-space, and have given some reasons for thinking that no such fibering is possible. The purpose of this note is to provide further evidence for this belief by proving that at least there is no compact fibering of the plane. The theorem I shall prove is in fact somewhat stronger.

THEOREM 1. If $f\left(E^{2}\right)=A$ is an interior ${ }^{2}$ transformation of the plane such that for each two points, $x$ and $y$, of $A, f^{-1}(x)$ and $f^{-1}(y)$ are homeomorphic, and such that each component of $f^{-1}(x)$ is compact, then no component of $f^{-1}(x)$ separates $E^{2}$ and $A$ is a 2-manifold.

If in addition $f^{-1}(x)$ is compact, then $f$ is monotone and $A$ is a plane.

PRoof. It cannot have escaped notice that a transformation satisfying the conditions placed on $f$ (omitting the homeomorphy condition) can be factored into a monotone closed transformation, $g\left(E^{2}\right)=B$, followed by a light interior transformation, $h(B)=A$, even though such theorems have been stated for compact spaces only. The arguments for Theorems ${ }^{3}$ VIII, 4.1, and VIII, 3.1, establish this. The proof of Theorem IX, 2.3, shows that $B$ is homeomorphic to a set obtained by removing some non-cut point from a cactoid. If some component of some $f^{-1}(x)$ separates $E^{2}$, then $B$ has a cut point. Since each set $g^{-1}(x)$ is a component of some set $f^{-1}(y), y$ in $A$, and each two inverses of points of $A$ are homeomorphic, $B$ has uncountably many cut points. Hence some point $p$ of $B$ is of Menger order 2, by Theorem VII, 3.2. There is a point $q$ of $B$ such that the closure, $C$, of the component of $B-q$ that contains $p$ is compact. Over $C, h$ is continuous, and over $C-q, h$ is interior; indeed, $h(C-q)$ is open in $A$.

Presented to the Society, December 27, 1946; received by the editors November $14,1946$.

1 Numbers in brackets refer to the bibliography.

2 A continuous transformation $f(X)=Y$ is interior provided that the image of every open subset of $X$ is open in $Y$, and is light provided that every set $f^{-1}(y), y$ in $Y$, is totally disconnected.

The statement of this theorem is weaker than that in Bull. Amer. Math. Soc. Abstract 53-1-106. In the original formulation, I used a characterization of the possible interior images of a 2-manifold that I had announced in Bull. Amer. Math. Soc. Abstract 52-5-220, but in the proof of which an error has been found.

3 Theorems referred to in this way are from Whyburn's book [4]. The roman numeral is the chapter number. 
Let $R$ be a neighborhood of $p$ whose boundary consists of just two points, and which lies with its boundary in $C-q$. By Theorem VIII, $7.3, \overline{h(R)}-h(R)$ is contained in $h(\bar{R}-R)$. Since $h(R)$ is open in $A$, it follows that its boundary is contained in $h(\bar{R}-R)$ and is therefore finite. Hence $A$ contains a point $x$ which separates some connected open subset, $D$, of $A$ into two separated sets, $D_{1}$ and $D_{2}$. Let $x_{1}$ and $x_{2}$ be points of $D_{1}$ and $D_{2}$, respectively. Let $K$ be a component of $f^{-1}(x)$; $K$ must separate $E^{2}$, for suppose that it does not. Then by Theorem VI, 3.11, in $f^{-1}(D)$ there is a 2-cell, $C$, that contains $K$, but has no point of $f^{-1}(x)$ in its boundary, and does not have any point of $f^{-1}\left(x_{1}+x_{2}\right)$ as limit point. Since $f(\bar{C})$ is in $D-\left(x_{1}+x_{2}\right)$, the boundary of $f(C)$ intersects both $D_{1}$ and $D_{2}$, and, by Theorem VIII, 7.3, is contained in the image of the boundary of $C$. But the image of the boundary of $C$ is a connected subset of $D-x$, and so lies wholly in $D_{1}$ or $D_{2}$. This is impossible, so that $K$ does separate $E^{2}$. In passing, notice that this implies that every component of each set $f^{-1}(y), y$ in $A$, separates $E^{2}$, by the hypothesis that each two point-inverses are homeomorphic. One component, $E$, of $E^{2}-K$ is bounded. Since $K+E$ is compact, over $K+E, f$ may be factored into a monotone transformation, $m$, followed by a light transformation, from Theorem VIII, 4.1. The continuum $m(K+E)$ has at least two non-cut points, one of which is $m(K)$; let $y$ be any other non-cut point. Then by Theorem VIII, 2.2, $m^{-1}(y)$ cannot separate $K+E$, so cannot separate $E$, and hence does not separate $E^{2}$. This contradiction shows that $B$ is a plane, so that $h(B)$ is a 2 -manifold, by Theorem $\mathrm{X}, 4.4$.

It remains to show that if each set $f^{-1}(x)$ is compact, then $f$ is monotone. This is true if the factor $h$ of the previous paragraph is a homeomorphism, so that the result will be established if $f$ is assumed light and proved to be a homeomorphism. Whyburn's Theorem X, 5.1, shows that a light interior transformation on a 2-manifold is locally finite-to-1. Hence there is an integer $k$ such that for each point $x$ of $A, f^{-1}(x)$ consists of exactly $k$ points. Adjoin a point $\omega$ to $E^{2}$ to make it a 2 -sphere, $S^{2}$. The collection $G$ whose elements are $\omega$ and all sets $f^{-1}(x)$ is continuous, for if the sequence $\left\{y_{n}\right\}$ of points of $A$ converges to the point $y$ of $A$, then by Theorem VII, 4.32the Eilenberg condition for interiority- $\left\{f^{-1}\left(y_{n}\right)\right\}$ converges to $f^{-1}(y)$ in $E^{2}$. The $k$-to-1 property implies that $\left\{f^{-1}\left(y_{n}\right)\right\}$ converges in $S^{2}$ to $f^{-1}(y)$. The continuity of $G$ means that $f$ can be extended to a light interior transformation, $g\left(S^{2}\right)=A^{\prime}$, which will necessarily be a local homeomorphism except at $\omega$. On page 197 of [4] Whyburn has noted that since an interior transformation on a locally connected compact continuum cannot raise coherence, $A^{\prime}$ is' either a 
sphere, a projective plane, or a closed 2-cell. The last of these is impossible under a local homeomorphism. In Theorem $\mathrm{X}, 7.3$, Whyburn has given a formula connecting the Euler characteristic of a compact 2-manifold with that of its image under a light interior transformation, $t$. Since $A^{\prime}$ has no boundary, this reduces here to

$$
k \chi\left(A^{\prime}\right)-\chi\left(S^{2}\right)=k r-n,
$$

where $r$ is the number of points $y$ in $A^{\prime}$ such that $t$ is not a local homeomorphism at some point in $t^{-1}(y)$ and $n$ is the total number of points in the inverses of all such points. For $t=g, r=n=1$; and $\chi\left(A^{\prime}\right)$ is either 1 or 2 . Hence either $k-2=k-1$, which is impossible, or $2 k-2$ $=k-1$, which implies that $k=1$, and that $g$ is a homeomorphism.

COROLlaRY 1. There is no compact fibering of the plane other than a homeomorphism.

Proof. Since a compact fibering, $f\left(E^{2}\right)=A$, satisfies all the hypotheses of Theorem 1 , it is necessarily monotone, and $A$ is a plane. Let $C$ be an open 2-cell in $A$, and let $F$ be a fiber. Then $f^{-1}(C)$ is homeomorphic to $C \times F$, if $C$ is sufficiently small. If $F$ is not a point, this implies that $f^{-1}(C)$ is the sum of uncountably many mutually exclusive open 2-cells, an impossibility in $E^{2}$.

I do not know of an example of an interior mapping of the plane onto a 2-manifold where each component of the inverse of each point is compact, but where the transformation is not light. However, if the hypotheses of the above theorem are strengthened by local connectivity assumptions, lightness can be proved.

COROLLARY 2. If, in addition to the hypotheses of the first part of Theorem 1, each component of each set $f^{-1}(x)$ is locally connected, then $f$ is light.

Proof. By Theorem 1, no component of a set $f^{-1}(x)$ separates $E^{2}$, so that no component is a simple closed curve. Hence every such component is either a point, an arc, or contains a simple triod. If one component of one point-inverse contained a simple triod, the condition that each two point-inverses are homeomorphic would establish the existence of an uncountable number of mutually exclusive triods in the plane; this would contradict Theorem 75, p. 254, of [5]. Hence each component of a point-inverse is either an arc or a point. Let $T$ be a nondegenerate component. In the notation of the first paragraph of the proof of Theorem 1 , since $B$ is a plane, and $h$ is interior, there exist an integer $k$ and a neighborhood $R$ of $g(T)$ such that $h(R)$ is at most $k$-to-1, by Theorem X, 5.1. It is easy to see that the Eilenberg 
condition for interiority implies that there is a point $z$ of $R-g(T)$ such that $g^{-1}(z)$ is an arc, and such that $h$ is locally 1-to-1 in a neighborhood $U$ of $z$; $U$ may be chosen to be simply connected and so small that each point of $U$ has a nondegenerate inverse under $g$. Then $g^{-1}(U)$ is open and simply connected. The collection of all sets $g^{-1}(x)$, $x$ in $U$, is a continuous collection of arcs filling up a set homeomorphic to a plane. J. H. Roberts has proved in [3] that there is not even an upper semi-continuous collection of arcs filling up a plane. Hence $f$ is light.

It is not difficult to see that the arguments for Theorem 1 and Corollary 2 can be combined and extended to prove the following.

Theorem 2. An interior transformation of a 2-manifold onto a 2manifold such that each component of each point-inverse is an arc or a point is necessarily light.

The mapping of the plane onto a cylinder of radius 1 obtained by identifying all vertical lines whose abscissae are congruent modulo $2 \pi$, and the mapping $w=\sin z$ are interior mappings of $E^{2}$ for which any two point-inverses are homeomorphic, showing that compactness is necessary in the second part of the theorem. If the requirement of interiority is removed instead, the conclusion of the second part is also false, as the following indication of an example ${ }^{4}$ shows: In the Cartesian plane, form undercut steps by making a downward crease along each line $x= \pm 1, \pm 4, \cdots, \pm(3 n+1), \cdots$, and by making an upward crease along each line $x= \pm 2, \pm 5, \cdots, \pm(3 n+2), \cdots$. "Flatten" the plane along these creases onto a plane $A$ so that each point of $A$ is covered by exactly 3 points of the original plane. I should remark that Civin has proved in [1] that no exactly 2-to-1, closed transformation of $E^{1}, E^{2}$, or $E^{3}$ exists.

\section{BIBLIOGRAPHY}

1. Paul Civin, Two-to-one mappings of manifolds, Duke Math. J. vol. 10 (1943) pp. $49-57$.

2. Deane Montgomery and Hans Samelson, Fiberings with singularities, Duke Math. J. vol. 13 (1946) pp. 51-56.

3. J. H. Roberts, Collections filling a plane, Duke Math. J. vol. 2 (1936) pp. 10-19.

4. G. T. Whyburn, Analytic topology, Amer. Math. Soc. Colloquium Publications, vol. 28.

5. R. L. Moore, Foundations of point set theory, Amer. Math. Soc. Colloquium Publications, vol. 13.

Purdue University

${ }^{4}$ This is modified from an example of a 3-to-1 mapping of an arc onto an arc due to Schweigert. 